\title{
Explosive growth of facet joint interventions in the medicare population in the United States: a comparative evaluation of 1997, 2002, and 2006 data
}

Laxmaiah Manchikanti ${ }^{1 *}$, Vidyasagar Pampati ${ }^{1}$, Vijay Singh², Mark V Boswell ${ }^{3}$, Howard S Smith ${ }^{4}$, Joshua A Hirsch ${ }^{5}$

\begin{abstract}
Background: The Office of Inspector General of the Department of Health and Human Services (OIG-DHHS) issued a report which showed explosive growth and also raised questions of lack of medical necessity and/or indications for facet joint injection services in 2006.

The purpose of the study was to determine trends of frequency and cost of facet joint interventions in managing spinal pain.
\end{abstract}

Methods: This analysis was performed to determine trends of frequency and cost of facet joint Interventions in managing spinal pain, utilizing the annual 5\% national sample of the Centers for Medicare and Medicaid Services (CMS) for 1997, 2002, and 2006.

Outcome measures included overall characteristics of Medicare beneficiaries receiving facet joint interventions, utilization of facet joint interventions by place of service, by specialty, reimbursement characteristics, and other variables.

Results: From 1997 to 2006, the number of patients receiving facet joint interventions per 100,000 Medicare population increased 386\%, facet joint visits increased $446 \%$, and facet joint interventions increased 543\%. The increases were higher in patients aged less than 65 years compared to those 65 or older with patients increasing $504 \%$ vs. $355 \%$, visits increasing $587 \%$ vs. $404 \%$, and services increasing $683 \%$ vs. $498 \%$.

Total expenditures for facet joint interventions in the Medicare population increased from over \$229 million in 2002 to over $\$ 511$ million in 2006 , with an overall increase of $123 \%$. In 2006, there was a 26.8-fold difference in utilization of facet joint intervention services in Florida compared to the state with the lowest utilization - Hawaii. There was an annual increase of $277.3 \%$ in the utilization of facet joint interventions by general physicians, whereas a $99.5 \%$ annual increase was seen for nurse practitioners (NPs) and certified registered nurse anesthetists (CRNAs) from 2002 to 2006. Further, in Florida, 47\% of facet joint interventions were performed by general physicians.

Conclusions: The reported explosive growth of facet joint interventions in managing spinal pain in certain regions and by certain specialties may result in increased regulations and scrutiny with reduced access.

\section{Background}

The Office of Inspector General (OIG)of the Department of Health and Human Services (OIG-DHHS), issued a report in September 2008 [1] noting that Medicare paid over $\$ 2$ billion in 2006 for interventional pain management (IPM) procedures. This report also showed

\footnotetext{
* Correspondence: drlm@thepainmd.com

'Pain Management Center of Paducah, 2831 Lone Oak Road, Paducah, KY,
} 42003, USA that Medicare payments for facet joint injections increased from \$141 million in 2003 to \$307 million in 2006. Of concern, $63 \%$ of facet joint injection services allowed by Medicare in 2006 did not meet the Centers for Medicare and Medicaid Services (CMS) program requirements, resulting in approximately $\$ 129$ million in improper payments. This report illustrated that facet joint injection services provided in an office were more likely to have an error than those provided in an 
ambulatory surgery center (ASC) or hospital outpatient department (HOPD). The OIG report also illustrated that approximately $35 \%$ of the Medicare facet joint injections were performed by non-interventional pain physicians. The OIG report recommended some radical changes in monitoring utilization of interventional techniques. Further, independent investigators also have shown an exponential increase in the performance of facet joint interventions [2-5].

Friedly et al $[3,6]$ reviewed trends in injection procedures focusing mainly on epidural injections from 1994 to 2001. Manchikanti et al [2] analyzed the growth of all interventional techniques in managing chronic pain in Medicare beneficiaries from 1997 to 2006. Both investigators demonstrated an overall increase of interventional techniques in all settings and in all parts of the country. The increase in the number of patients receiving IPM services per 100,000 of Medicare recipients was 137\% with an overall increase of IPM services of $197 \%$ per 100,000 Medicare beneficiaries increasing by $197 \%$. However, the most dramatic increase was found to be for facet joint interventions with a 543\% increase per 100,000 Medicare beneficiaries.

Chronic spinal pain in the United States is highly prevalent with substantial economic impact [7-16]. However, the treatment of spinal pain is controversial, in part related to the wide variability in the treatments utilized [16]. The rising prevalence of chronic low back pain has been demonstrated with continued high levels of disability and health care use [7]. Freburger et al [7] showed an annual increase of $11.6 \%$ of chronic low back pain and attributed a substantial portion of rising low back pain care costs over the past 2 decades to this rising prevalence. Chronic spinal pain is associated with functional and psychological disabilities and health, social, and economic impact, especially in the elderly [10-13,17,18].

Epidural injections and facet joint interventions are the 2 most commonly utilized procedures in IPM $[1-6,19,20]$. However, the literature addressing the effectiveness of facet joint interventions, though emerging, is highly variable, based on the technique, outcome measures, patient selection, and methodology [21-28].

Health care spending in the United States is escalating and the long-range fiscal sustainability of Medicare is in question [26-29]. In a report titled Accounting for the Cost of US Health Care: A New Look at Why Americans Spend More [30] it was found that in 2006 the United States spent $\$ 650$ billion more on health care than any of its peer Organisation for Economic Co-operation and Development (OECD) countries, even after adjusting for wealth. The majority of the excess spending was derived from outpatient care. One of the means of controlling health care expenditures is by ensuring that all care is medically necessary and avoiding overuse, abuse, and fraud. The OIG report suggests that there is significant overuse, abuse, and potential fraud in performing facet joint interventions in the United States.

In this study, we sought to evaluate the use of all types of facet joint interventions (i.e., intraarticular injections, facet joint nerve blocks, and facet joint neurotomy) in the lumbar, cervical, and thoracic spine. In addition, our purpose was to identify trends in the number of procedures, reimbursement, specialty involvement, fluoroscopy use, and indications. Finally, we sought to explore the association between overall injection costs and the volume of services provided in HOPD settings, ACSs, and in-office settings.

\section{Methods}

The data for this study was used from the standard 5\% national sample of the CMS physician outpatient billing claims for 1997, 2002, and 2006. The data set is a sample of those enrolled in the fee-for-service Medicare program based on selecting records with specific numbers in positions 8 and 9 of the health insurance claim number and is generated by CMS. The CMS 5\% sample data set is therefore unbiased and unpredictable in terms of any patient characteristics, but does allow appropriate tracking of patients over time and across databases. Consequently, CMS makes this 5\% sample available to researchers. In addition, a $100 \%$ data set is so large that it is not feasible to use for research purposes. Thus, Institutional Review Board (IRB) approval was not required. CMS's providing the data also does not require IRB approval prior to analysis or publication.

Previous studies $[3,6]$ generally included patients aged 65 and older. We have studied all patients enrolled in Medicare who received interventional techniques [2]. Overall Medicare enrolled over 43 million beneficiaries in 2006, and is the single largest health care payor in the United States [31]. Consequently, the Medicare data set includes a large proportion of procedures for spinal pain being performed in the United States, including facet joint interventions. In addition to patient age, the database included the Current Procedural Terminology (CPT) procedure codes; the International Classification of Diseases, $9^{\text {th }}$ Revision, Clinical Modification (ICD-9$\mathrm{CM})$ diagnosis codes; date of service, provider specialty, provider zip code, and allowed charges.

To yield data for the entire beneficiary population of Medicare, results from the 5\% sample were multiplied by 20 . In addition, rates were calculated based on Medicare beneficiaries for the corresponding year and are reported as per 100,000 Medicare beneficiaries. The data were tabulated based on the place of service - HOPD, ASC, or office for the years 1997, 2002, and 2006. Facility charges were also identified for HOPDs, ASCs, and 
offices (office facility portion as overhead expense equals total office payment minus physician payment). Facility payments for HOPD were estimated based on national payment rates with consideration of modifiers, due to the non-availability of HOPD data in the data set. Allowed charges were used to estimate the costs of Medicare for these procedures and costs were adjusted for health care inflation using the U.S. Bureau of Labor Statistics Consumer Price Index (CPI) for medical care services and represent costs for 2006 [32].

In this study, all types of facet joint interventions with CPT codes 64470, 64472, 64475, 64476, 64622, 64623, 64626, and 64627, with evaluation of Medicare data of 1997, 2002, and 2006 were utilized. Appropriate considerations were given to the changes in the CPT with introduction of new codes or replacement codes.

In addition, diagnostic codes were utilized from the ICD-9-CM. The previous studies excluded cervical and thoracic facet joint interventions [3,6]; they argued that cervical and thoracic spine disorders differ clinically from lumbar spine disorders and may be the result of different disease processes. They believed that cervical and thoracic interventions represent a very small proportion of patients. However, the emerging statistics show that cervical and thoracic facet joint interventions occupy a large proportion of facet joint interventions. Thus, it was felt essential to include these interventions.

To analyze the data based on specialty, the IPM specialties were described as those providers designated in IPM -09, pain medicine -72, anesthesiology -05, physical medicine and rehabilitation -25 , neurology -13 , psychiatry -26 , orthopedic surgery -20 , and neurosurgery -14 [33]. General practitioners -01, family practitioners -08, and internists -11 were considered as general physicians. All other providers were considered as other physicians and providers.

\section{Data Synthesis}

The data were analyzed using SPSS (9.0) statistical software, Microsoft Access 2003, and Microsoft Excel (2003). The procedure rates were calculated per 100,000 Medicare beneficiaries.

\section{Results}

\section{Population Characteristics}

Table 1 illustrates the characteristics of Medicare beneficiaries and facet joint interventions. During the same period, Medicare recipients receiving facet joint interventions increased $386 \%$. Facet joint interventions increased from 606 per 100,000 in 1997 to 3,895 per 100,000 in 2006 , a $543 \%$ increase.

The results illustrate a higher proportion of increase for patients under 65; that proportion of patients increased $504 \%$ vs. $355 \%$. For those 65 or over, visits increased $404 \%$ versus $587 \%$ for those under 65 ; services for those over 65 increased $498 \%$ versus $683 \%$ for those under 65 . The Medicare population below the age of 65 years increased $45.4 \%$ in contrast to $8 \%$ of those 65 years or older.

\section{Utilization Characteristics}

Table 2 illustrates the summary of frequency of utilization of facet joint interventions based on CPT code and place of service. Due to the 1997 data being non-comparable and not comprehensive, the data from 2002 and 2006 were utilized. The majority of the procedures $(80 \%$ in 2002 and $77 \%$ in 2006) were performed in the lumbar region, with cervical and thoracic procedures constituting $20 \%$ in 2002 and $23 \%$ in 2006 . The most commonly performed procedure was subsequent lumbar facet joint injection/nerve block (CPT 64476). Cervical/thoracic interventions increased 194\% per 100,000 Medicare beneficiaries, whereas lumbar procedures increased $151 \%$. In $2002,40 \%$ of procedures were performed in HOPD settings and $41.7 \%$ in office settings; whereas in 2006, $59.6 \%$ were performed in office settings. The overall rate (per 100,000 Medicare beneficiaries) increased by $160 \%$ from 2002 to 2006; whereas in office settings the rate increased significantly (271\%), followed by ASCs (168\%) and HOPD settings (40\%). Cervical procedures increased $194 \%$ with a distribution of $259 \%, 224 \%$, and $59 \%$ in office, ASC, and HOPD settings.

\section{Reimbursement Characteristics}

Additional file 1 illustrates physician and facility reimbursement by place of service adjusted for inflation for years 2002 and 2006. As seen in Additional file 1, overall facility average charges decreased by $26 \%$.

\section{Specialty Characteristics}

Figure 1 illustrates the increase in utilization of facet joint interventions by various specialty groups assigned as IPM, general practice, NPs/CRNAs, and others from 2002 to 2006. Across the country, the majority of procedures were performed by IPM physicians with $87 \%$ in 2002 and $74.5 \%$ in 2006. However, in 2006 general physicians performed $18.6 \%$ of these procedures, while all others performed $6.9 \%$ of the procedures (Table 3 ). Overall increases were greatest for general physicians, increasing by over 1,109\% from 2002 to 2006, an annual growth of $277.3 \%$. There was also an increase of $398 \%$ from 2002 to 2006 among NPs and CRNAs, an annual increase of $99.5 \%$. In Florida in 2006, $47 \%$ of the procedures were performed by general physicians with specialties of general practice, family practice, and internal medicine. 
Table 1 Characteristics of Medicare beneficiaries and facet joint interventions.

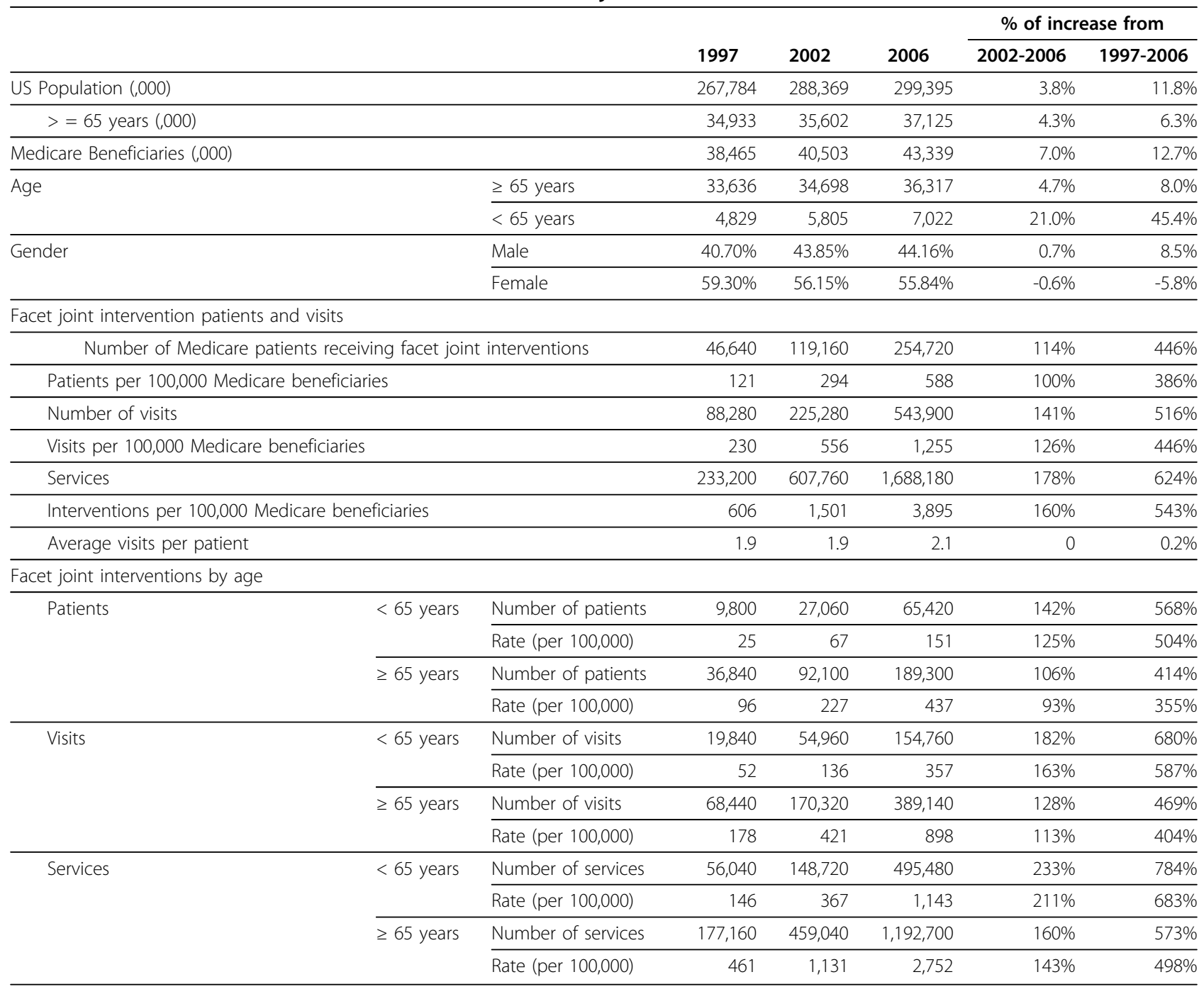

\section{Fluoroscopy Utilization}

Figure 2 illustrates fluoroscopy utilization based on specialty. Overall in 2002, $48 \%$ of all visits included fluoroscopy, compared to $63 \%$ visits of all visits in 2006 .

\section{Procedural Characteristics by State}

Table 4 illustrates facet joint interventions for each state. South Dakota showed the highest increase of $504 \%$ with Alabama showing the lowest increase of $14 \%$ per 100,000 Medicare beneficiaries. The overall increase for the United States was 160\% from 2002 to 2006. However, smaller states with a small number of procedures, such as South Dakota, preclude any conclusions to be drawn as per the increases. Thus, when normalized for population, Florida showed a 26.8-fold difference from Hawaii, the state with the lowest, for 2006. All other states showed a difference of less than 10-fold with Michigan showing a 9.87-fold difference, Texas showing an 8.42-fold difference, Arkansas showing a 7.34-fold difference, and Delaware showing a 6.47-fold difference, compared to the lowest state for 2006. Further, facet joint procedures per state as a proportion of national utilization declined in multiple states.

\section{Diagnostic Characteristics}

Table 5 illustrates the utilization of ICD-9-CM diagnostic codes for facet joint interventions. The most common diagnoses documented were "lumbosacral spondylosis" in the lumbar spine of $32.3 \%$ and cervical spondylosis in the cervical spine of $5.3 \%$. Degenerative disc disease was the diagnosis criteria utilized in $6.2 \%$ and $1.2 \%$ of cases in the lumbar and cervical spine respectively. Thus, accurate diagnosis was utilized in fewer than $50 \%$ of patients in 2006 . 
Table 2 Utilization of facet joint interventions by place of service.

\begin{tabular}{|c|c|c|c|c|c|c|c|c|c|c|c|c|}
\hline \multirow[t]{3}{*}{ CPT } & \multicolumn{4}{|c|}{2002} & \multicolumn{4}{|c|}{2006} & \multicolumn{4}{|c|}{ Change from 2002} \\
\hline & \multicolumn{4}{|c|}{ Place of Service } & \multicolumn{4}{|c|}{ Place of Service } & \multicolumn{4}{|c|}{ Place of Service } \\
\hline & ASC & HOPD & Office & Total & ASC & HOPD & Office & Total & ASC & HOPD & Office & Total \\
\hline \multicolumn{13}{|c|}{ Cervical/Thoracic (C/T) } \\
\hline 64470 & 6,100 & 10,220 & 26,320 & 42,640 & 18,520 & 17,300 & 89,300 & 125,120 & $204 \%$ & $69 \%$ & $239 \%$ & $193 \%$ \\
\hline 64472 & 10,380 & 19,380 & 34,360 & 64,120 & 34,340 & 32,300 & 145,400 & 212,040 & $231 \%$ & $67 \%$ & $323 \%$ & $231 \%$ \\
\hline $64470-72$ & 16,480 & 29,600 & 60,680 & 106,760 & 52,860 & 49,600 & 234,700 & 337,160 & $221 \%$ & $68 \%$ & $287 \%$ & $216 \%$ \\
\hline Rate & 41 & 73 & 150 & 264 & 122 & 114 & 542 & 778 & $200 \%$ & $57 \%$ & $261 \%$ & $195 \%$ \\
\hline 64626 & 1,020 & 2,280 & 1,400 & 4,700 & 4,700 & 3,580 & 5,340 & 13,620 & $361 \%$ & $57 \%$ & $281 \%$ & $190 \%$ \\
\hline 64627 & 2,120 & 4,160 & 3,760 & 10,040 & 10,360 & 8,180 & 12,800 & 31,340 & $389 \%$ & $97 \%$ & $240 \%$ & $212 \%$ \\
\hline $64626-27$ & 3,140 & 6,440 & 5,160 & 14,740 & 15,060 & 11,760 & 18,140 & 44,960 & $380 \%$ & $83 \%$ & $252 \%$ & $205 \%$ \\
\hline Rate & 8 & 16 & 13 & 36 & 35 & 27 & 42 & 104 & $348 \%$ & $71 \%$ & $229 \%$ & $185 \%$ \\
\hline $\mathrm{C} / \mathrm{T}$ Total & 19,620 & 36,040 & 65,840 & 121,500 & 67,920 & 61,360 & 252,840 & 382,120 & $246 \%$ & $70 \%$ & $284 \%$ & $215 \%$ \\
\hline Rate & 48 & 89 & 163 & 300 & 157 & 142 & 583 & 882 & $224 \%$ & $59 \%$ & $259 \%$ & $194 \%$ \\
\hline \multicolumn{13}{|c|}{ Lumbar/Sacral (L/S) } \\
\hline 64475 & 26,120 & 60,340 & 69,960 & 156,420 & 67,580 & 84,420 & 214,160 & 366,160 & $159 \%$ & $40 \%$ & $206 \%$ & $134 \%$ \\
\hline 64476 & 47,300 & 101,560 & 93,680 & 242,540 & 114,400 & 143,040 & 375,980 & 633,420 & $142 \%$ & $41 \%$ & $301 \%$ & $161 \%$ \\
\hline $64475-76$ & 73,420 & 161,900 & 163,640 & 398,960 & 181,980 & 227,460 & 590,140 & 999,580 & $148 \%$ & $40 \%$ & $261 \%$ & $151 \%$ \\
\hline Rate & 181 & 400 & 404 & 985 & 420 & 525 & 1,362 & 2,306 & $132 \%$ & $31 \%$ & $237 \%$ & $134 \%$ \\
\hline 64622 & 5,420 & 13,360 & 6,660 & 25,440 & 20,400 & 22,880 & 37,780 & 81,060 & $276 \%$ & $71 \%$ & $467 \%$ & $219 \%$ \\
\hline 64623 & 12,660 & 31,660 & 17,540 & 61,860 & 47,940 & 51,840 & 125,640 & 225,420 & $279 \%$ & $64 \%$ & $616 \%$ & $264 \%$ \\
\hline $64622-23$ & 18,080 & 45,020 & 24,200 & 87,300 & 68,340 & 74,720 & 163,420 & 306,480 & $278 \%$ & $66 \%$ & $575 \%$ & $251 \%$ \\
\hline Rate & 45 & 111 & 60 & 216 & 158 & 172 & 377 & 707 & $253 \%$ & $55 \%$ & $531 \%$ & $228 \%$ \\
\hline L/S Total & 91500 & 206,920 & 187,840 & 486,260 & 250,320 & 302,180 & 753,560 & $1,306,060$ & $174 \%$ & $46 \%$ & $301 \%$ & $169 \%$ \\
\hline Rate & 226 & 511 & 464 & 1,201 & 578 & 697 & 1,739 & 3,014 & 156 & $36 \%$ & $275 \%$ & $151 \%$ \\
\hline \multicolumn{13}{|c|}{ Grand Total } \\
\hline Services & 111,120 & 242,960 & 253,680 & 607,760 & 318,240 & 363,540 & $1,006,400$ & $1,688,180$ & $186 \%$ & $50 \%$ & $297 \%$ & $178 \%$ \\
\hline Rate & 274 & 600 & 626 & 1,501 & 734 & 839 & 2,322 & 3,895 & $168 \%$ & $40 \%$ & $271 \%$ & $160 \%$ \\
\hline
\end{tabular}

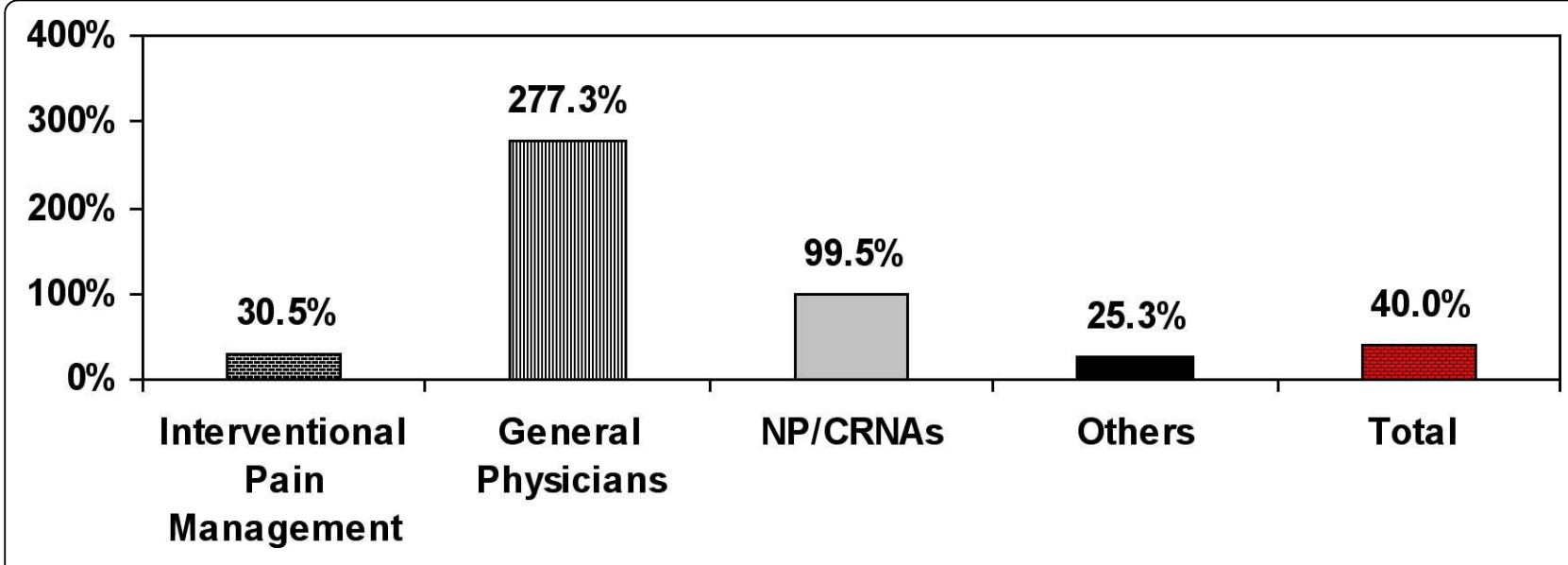

Figure 1 Annual percentage of increase of facet joint intervention services per 100,000 Medicare recipients from 2002 to 2006. 
Table 3 Utilization of facet joint interventions by speciality.

\begin{tabular}{|c|c|c|c|c|c|c|c|c|}
\hline \multirow[b]{2}{*}{ Speciality } & \multicolumn{3}{|c|}{2002} & \multicolumn{3}{|c|}{2006} & \multicolumn{2}{|c|}{ Change from 2002} \\
\hline & Services & Percent & Rate & Services & Percent & Rate & Percent & Rate \\
\hline Interventional Pain Management & 529,220 & $87.1 \%$ & 1,307 & $1,256,860$ & $74.5 \%$ & 2,900 & $-15 \%$ & $122 \%$ \\
\hline Anesthesiology & 338,660 & $55.7 \%$ & 836 & 524,340 & $31.1 \%$ & 1,210 & $-44 \%$ & $45 \%$ \\
\hline Pain Management & 78,080 & $12.8 \%$ & 193 & 459,520 & $27.2 \%$ & 1,060 & $112 \%$ & $450 \%$ \\
\hline Anesthesiology \& Pain Management & 416,740 & $68.5 \%$ & 1,029 & 983,860 & $58.3 \%$ & 2,270 & $-15 \%$ & $121 \%$ \\
\hline Physical Medicine and Rehabilitation & 54,000 & $8.9 \%$ & 133 & 148,980 & $8.8 \%$ & 344 & $-1 \%$ & $158 \%$ \\
\hline Orthopedic Surgery & 24,600 & $4.0 \%$ & 61 & 51,860 & $3.1 \%$ & 120 & $-24 \%$ & $97 \%$ \\
\hline Neurology & 23,140 & $3.8 \%$ & 57 & 49,400 & $2.9 \%$ & 114 & $-23 \%$ & $100 \%$ \\
\hline Neurosurgery & 9,320 & $1.5 \%$ & 23 & 21,080 & $1.2 \%$ & 49 & $-19 \%$ & $111 \%$ \\
\hline Psychiatry & 1,420 & $0.2 \%$ & 4 & 1,680 & $0.1 \%$ & 4 & $-57 \%$ & $11 \%$ \\
\hline Family \& General Practice/Internal Medicine & 24,300 & $4.0 \%$ & 60 & 314,420 & $18.6 \%$ & 725 & $366 \%$ & $1109 \%$ \\
\hline Others & 54,240 & $8.9 \%$ & 134 & 116,900 & $6.9 \%$ & 270 & $-22 \%$ & $101 \%$ \\
\hline Diagnostic Radiology & 14,100 & $2.3 \%$ & 35 & 20,140 & $1.2 \%$ & 46 & $-49 \%$ & $33 \%$ \\
\hline Nurse Practitioners/CRNA's & 860 & $0.1 \%$ & 2 & 4,580 & $0.3 \%$ & 11 & $92 \%$ & $398 \%$ \\
\hline Others & 39,280 & $6.5 \%$ & 97 & 92,180 & $5.5 \%$ & 213 & $-16 \%$ & $119 \%$ \\
\hline Total & 607,760 & $100 \%$ & 1,501 & $1,688,180$ & $100 \%$ & 3,895 & $178 \%$ & $160 \%$ \\
\hline
\end{tabular}

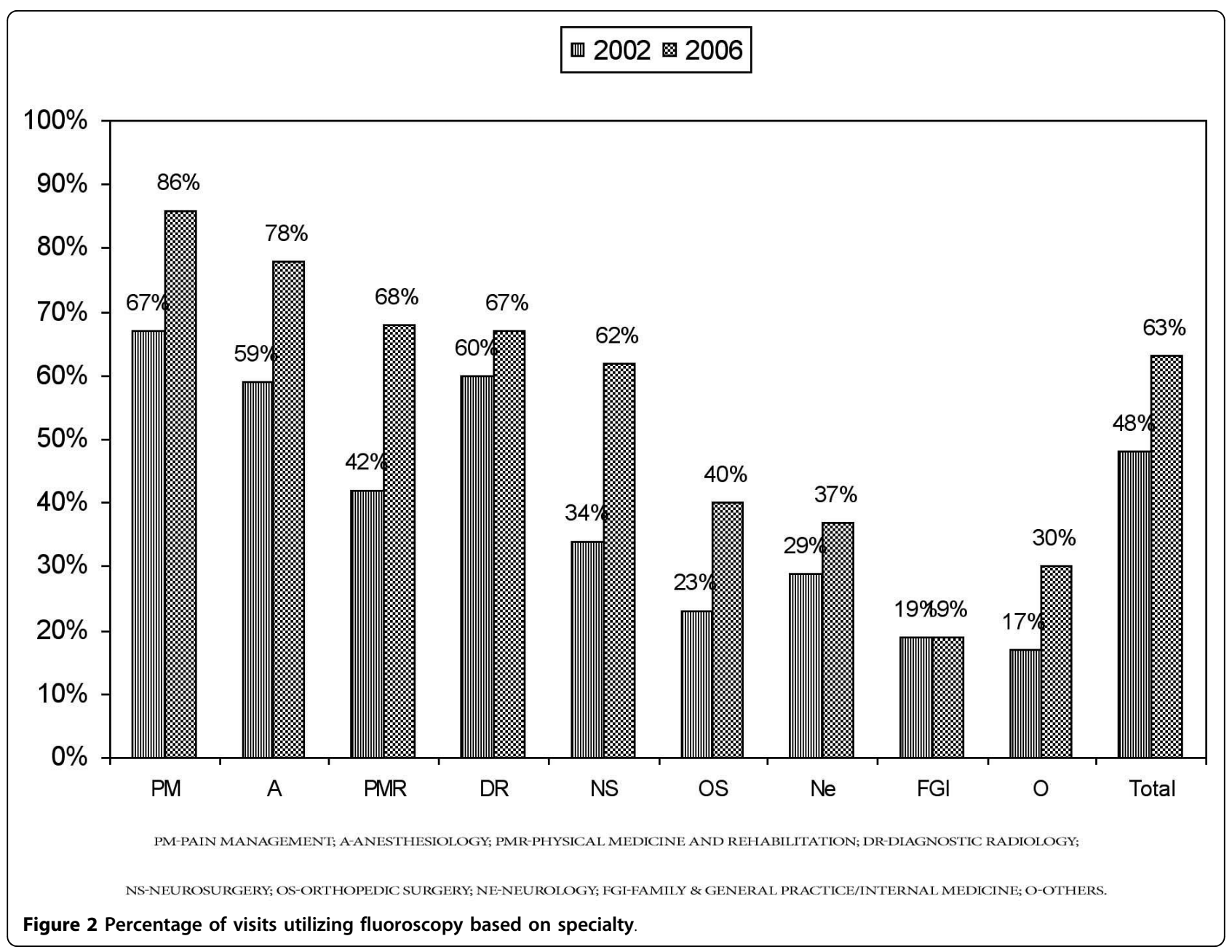


Table 4 Number of facet joint interventions and procedures per 100,000 Medicare beneficiaries provided by state.

\begin{tabular}{|c|c|c|c|c|c|c|c|}
\hline \multirow[b]{2}{*}{ State } & \multicolumn{2}{|l|}{2002} & \multicolumn{2}{|l|}{2006} & \multicolumn{2}{|c|}{$\%$ of change from 2002} & \multirow{2}{*}{$\begin{array}{l}\text { Fold difference from the } \\
\text { lowest state for } 2006\end{array}$} \\
\hline & Services & $\begin{array}{l}\text { Rate per } 100,000 \\
\text { population }\end{array}$ & Services & $\begin{array}{l}\text { Rate per } 100,000 \\
\text { population }\end{array}$ & Services & $\begin{array}{l}\text { Rate per } 100,000 \\
\text { population }\end{array}$ & \\
\hline Florida & 108,800 & 3,603 & 534,000 & 17,340 & $391 \%$ & $381 \%$ & 26.80 \\
\hline Michigan & 44,940 & 3,514 & 96,460 & 6,386 & $115 \%$ & $82 \%$ & 9.87 \\
\hline Texas & 62,680 & 2,680 & 142,960 & 5,445 & $128 \%$ & $103 \%$ & 8.42 \\
\hline Arkansas & 8,240 & 1,692 & 23,040 & 4,752 & $180 \%$ & $181 \%$ & 7.34 \\
\hline Delaware & 800 & 714 & 5,520 & 4,187 & $590 \%$ & $486 \%$ & 6.47 \\
\hline Alaska & 400 & 874 & 2,000 & 4,026 & $400 \%$ & $361 \%$ & 6.22 \\
\hline Mississippi & 6,920 & 1,788 & 16,600 & 3,596 & $140 \%$ & $101 \%$ & 5.56 \\
\hline Kentucky & 11,520 & 1,797 & 24,900 & 3,583 & $116 \%$ & $99 \%$ & 5.54 \\
\hline$\overline{U t a h}$ & 2,620 & 1,365 & 8,440 & 3,431 & $222 \%$ & $151 \%$ & 5.30 \\
\hline Tennessee & 12,440 & 1,695 & 32,460 & 3,419 & $161 \%$ & $102 \%$ & 5.29 \\
\hline West Virginia & 3,160 & 878 & 12,080 & 3,343 & $282 \%$ & $281 \%$ & 5.17 \\
\hline Montana & 2,740 & 1,745 & 5,060 & 3,335 & $85 \%$ & $91 \%$ & 5.15 \\
\hline Maryland & 8,500 & 1,302 & 23,320 & 3,294 & $174 \%$ & $153 \%$ & 5.09 \\
\hline North Carolina & 15,840 & 1,331 & 42,400 & 3,218 & $168 \%$ & $142 \%$ & 4.97 \\
\hline Ohio & 17,620 & 1,134 & 56,060 & 3,153 & $218 \%$ & $178 \%$ & 4.87 \\
\hline Vermont & 800 & 875 & 2,900 & 3,150 & $263 \%$ & $260 \%$ & 4.87 \\
\hline South Carolina & 6,540 & 965 & 21,160 & 3,140 & $224 \%$ & $225 \%$ & 4.85 \\
\hline Missouri & 8,260 & 1,109 & 29,160 & 3,137 & $253 \%$ & $183 \%$ & 4.85 \\
\hline New Hampshire & 3,320 & 2,024 & 6,200 & 3,134 & $87 \%$ & $55 \%$ & 4.84 \\
\hline Alabama & 20,220 & 2,682 & 23,620 & 3,058 & $17 \%$ & $14 \%$ & 4.73 \\
\hline Indiana & 12,620 & 1,485 & 28,140 & 3,050 & $123 \%$ & $105 \%$ & 4.71 \\
\hline Pennsylvania & 31,560 & 1,552 & 63,740 & 2,957 & $102 \%$ & $90 \%$ & 4.57 \\
\hline Georgia & 14,820 & 1,705 & 31,360 & 2,916 & $112 \%$ & $71 \%$ & 4.51 \\
\hline South Dakota & 580 & 480 & 3,460 & 2,904 & $497 \%$ & $504 \%$ & 4.49 \\
\hline lowa & 7,780 & 1,784 & 13,960 & 2,823 & $79 \%$ & $58 \%$ & 4.36 \\
\hline Louisiana & 4,220 & 701 & 17,500 & 2,804 & $315 \%$ & $300 \%$ & 4.33 \\
\hline Arizona & 5,960 & 753 & 22,540 & 2,765 & $278 \%$ & $267 \%$ & 4.27 \\
\hline Wyoming & 780 & 1,158 & 1,780 & 2,593 & $128 \%$ & $124 \%$ & 4.01 \\
\hline Massachusetts & 10,280 & 1,155 & 25,240 & 2,571 & $146 \%$ & $123 \%$ & 3.97 \\
\hline California & 55,060 & 1,458 & 103,000 & 2,409 & $87 \%$ & $65 \%$ & 3.72 \\
\hline Wisconsin & 10,060 & 1,435 & 19,660 & 2,341 & $95 \%$ & $63 \%$ & 3.62 \\
\hline$\overline{\text { Maine }}$ & 2,640 & 1,153 & 5,560 & 2,311 & $111 \%$ & $100 \%$ & 3.57 \\
\hline New York & 27,660 & 1,057 & 63,840 & 2,276 & $131 \%$ & $115 \%$ & 3.52 \\
\hline New Mexico & 2,720 & 925 & 6,120 & 2,219 & $125 \%$ & $140 \%$ & 3.43 \\
\hline Kansas & 2,000 & 531 & 8,980 & 2,209 & $349 \%$ & $316 \%$ & 3.41 \\
\hline Illinois & 17,060 & 1,054 & 37,180 & 2,171 & $118 \%$ & $106 \%$ & 3.35 \\
\hline Nevada & 2,640 & 996 & 6,580 & 2,145 & $149 \%$ & $115 \%$ & 3.32 \\
\hline Virginia & 10,720 & 1,203 & 19,900 & 1,955 & $86 \%$ & $62 \%$ & 3.02 \\
\hline New Jersey & 13,320 & 1,073 & 23,180 & 1,867 & $74 \%$ & $74 \%$ & 2.89 \\
\hline Colorado & 4,740 & 946 & 10,020 & 1,856 & $111 \%$ & $96 \%$ & 2.87 \\
\hline Oklahoma & 5,920 & 1,159 & 10,260 & 1,854 & $73 \%$ & $60 \%$ & 2.86 \\
\hline Connecticut & 3,040 & 559 & 9,160 & 1,728 & $201 \%$ & $209 \%$ & 2.67 \\
\hline
\end{tabular}


Table 4: Number of facet joint interventions and procedures per 100,000 Medicare beneficiaries provided by state. (Continued)

\begin{tabular}{|c|c|c|c|c|c|c|c|}
\hline Minnesota & 3,440 & 587 & 11,940 & 1,674 & $247 \%$ & $185 \%$ & 2.59 \\
\hline Idaho & 1,760 & 1,019 & 3,100 & 1,656 & $76 \%$ & $63 \%$ & 2.56 \\
\hline Nebraska & 1,100 & 430 & 3,440 & 1,382 & $213 \%$ & $222 \%$ & 2.14 \\
\hline Washington & 4,560 & 667 & 11,560 & 1,365 & $154 \%$ & $105 \%$ & 2.11 \\
\hline Rhode Island & 880 & 511 & 2,060 & 1,332 & $134 \%$ & $161 \%$ & 2.06 \\
\hline Oregon & 1,440 & 295 & 7,240 & 1,310 & $403 \%$ & $344 \%$ & 2.02 \\
\hline North Dakota & 960 & 930 & 1,160 & 1,184 & $21 \%$ & $27 \%$ & 1.83 \\
\hline $\begin{array}{l}\text { District of } \\
\text { Columbia }\end{array}$ & 360 & 485 & 620 & 1,021 & $72 \%$ & $110 \%$ & 1.58 \\
\hline Hawaii & 720 & 420 & 1,100 & 647 & $53 \%$ & $54 \%$ & 1.00 \\
\hline Overall & 607,760 & 1,501 & $1,688,180$ & 3,895 & $178 \%$ & $160 \%$ & 6.02 \\
\hline
\end{tabular}

Table 5 Line of diagnosis for facet joint interventions.

\begin{tabular}{|c|c|c|c|c|}
\hline Group & 2002 & Percent & 2006 & Percent \\
\hline LUMBOSACRAL SPONDYLOSIS & 168,980 & $32.3 \%$ & $3,379,600$ & $32.3 \%$ \\
\hline LUMBAGO/BACK PAIN & 151,240 & $28.9 \%$ & $3,024,800$ & $28.9 \%$ \\
\hline CERVICAL SPONDYLOSIS W/WO MYELOPATHY & 27,960 & $5.3 \%$ & 559,200 & $5.3 \%$ \\
\hline DEGENERATION OF LUMBAR OR LUMBOSACRAL INTERVERTEBRAL DISC & 32,180 & $6.2 \%$ & 643,600 & $6.2 \%$ \\
\hline CERVICALGIA & 29,320 & $5.6 \%$ & 586,400 & $5.6 \%$ \\
\hline SCIATICA & 2,800 & $0.5 \%$ & 56,000 & $0.5 \%$ \\
\hline THORACIC OR LUMBOSACRAL NEURITIS OR RADICULITIS UNSPECIFIED & 21,680 & $4.1 \%$ & 433,600 & $4.1 \%$ \\
\hline THORACIC SPONDYLOSIS W/WO MYELOPATHY & 4,320 & $0.8 \%$ & 86,400 & $0.8 \%$ \\
\hline SPINAL STENOSIS & 11,940 & $2.3 \%$ & 238,800 & $2.3 \%$ \\
\hline POSTLAMINECTOMY SYNDROME & 10,860 & $2.1 \%$ & 217,200 & $2.1 \%$ \\
\hline DEGENERATION OF CERVICAL INTERVERTEBRAL DISC & 6,040 & $1.2 \%$ & 120,800 & $1.2 \%$ \\
\hline LUMBAR DISC DISPLACEMENT & 6,980 & $1.3 \%$ & 139,600 & $1.3 \%$ \\
\hline PAIN IN JOINT UNSPECIFIED/SPECIFIED AREA & 5,320 & $1.0 \%$ & 106,400 & $1.0 \%$ \\
\hline BRACHIAL NEURITIS OR RADICULITIS NOT OTHERWISE SPECIFIED & 4,560 & $0.9 \%$ & 91,200 & $0.9 \%$ \\
\hline ARTHROPATHY & 1,680 & $0.3 \%$ & 33,600 & $0.3 \%$ \\
\hline OTHER SYNDROMES AFFECTING CERVICAL REGION & 5,640 & $1.1 \%$ & 112,800 & $1.1 \%$ \\
\hline POSTLAMINECTOMY SYNDROME OF CERVICAL REGION & 1,000 & $0.2 \%$ & 20,000 & $0.2 \%$ \\
\hline LUMBOSACRAL SPRAIN & 1,680 & $0.3 \%$ & 33,600 & $0.3 \%$ \\
\hline DEGENERATION OF THORACIC OR THORACOLUMBAR INTERVERTEBRAL DISC & 1,220 & $0.2 \%$ & 24,400 & $0.2 \%$ \\
\hline CONGENITAL ANOMALIES OF SPINE & 1,200 & $0.2 \%$ & 24,000 & $0.2 \%$ \\
\hline DISORDERS OF SACRUM & 2,300 & $0.4 \%$ & 46,000 & $0.4 \%$ \\
\hline SPONDYLOLISTHESIS & 1,120 & $0.2 \%$ & 22,400 & $0.2 \%$ \\
\hline MYALGIA AND MYOSITIS & 1,560 & $0.3 \%$ & 31,200 & $0.3 \%$ \\
\hline DEGENERATION OF INTERVERTEBRAL DISC SITE UNSPECIFIED & 940 & $0.2 \%$ & 18,800 & $0.2 \%$ \\
\hline NEURALGIA NEURITIS AND RADICULITIS UNSPECIFIED & 520 & $0.1 \%$ & 10,400 & $0.1 \%$ \\
\hline OSTEOARTHROSIS & 1,180 & $0.2 \%$ & 23,600 & $0.2 \%$ \\
\hline SPINAL STENOSIS IN CERVICAL REGION & 800 & $0.2 \%$ & 16,000 & $0.2 \%$ \\
\hline SPASM OF MUSCLE & 840 & $0.2 \%$ & 16,800 & $0.2 \%$ \\
\hline PATHOLOGICAL FRACTURE OF VERTEBRAE & 400 & $0.1 \%$ & 8,000 & $0.1 \%$ \\
\hline LUMBOSACRAL PLEXUS LESIONS & 800 & $0.2 \%$ & 16,000 & $0.2 \%$ \\
\hline INFLAMMATORY SPONDYLOPATHY & 520 & $0.1 \%$ & 10,400 & $0.1 \%$ \\
\hline OTHERS & 12,840 & $2.5 \%$ & 256,800 & $2.5 \%$ \\
\hline
\end{tabular}




\section{Overall Growth Pattern}

Figure 3 illustrates the overall growth pattern of facet joint interventions. These annual rates of increase for facet joint interventions represent the years from 1997 to 2006. There was an increase of facet joint interventions by general physicians of over $1,109 \%$.

\section{Discussion}

Facet joint intervention rates for spinal disorders increased dramatically over the study period from 1997 to 2006. This increase per 100,000 Medicare population from 1997 to 2006 was relatively constant over time, resulting in an increase of facet joint patients of $386 \%$, facet joint visits of $446 \%$, and facet joint interventions of $543 \%$. Facet joint interventions also increased based on age. Among Medicare recipients per 100,000, less than 65 years of age, compared to those 65 or older, the patient population receiving facet joint interventions increased $504 \%$ vs. $355 \%$, visits increased $587 \%$ compared to $404 \%$, and services increased $683 \%$ compared to $498 \%$. In addition, total expenditures also increased from over $\$ 229$ million in 2002 to over $\$ 511$ million in 2006 , with an overall increase of $123 \%$ from 2002 to 2006. There was a significant increase of $1,109 \%$ in the utilization of facet joint interventions by general physicians - composed of general practice, family practice, and internal medicine - from 2002 to 2006, an annual increase of $277.3 \%$. There were also significant usage or utilization increases among NPs and CRNAs from 2002 to 2006 of $398 \%$, an annual increase of $99.5 \%$. These

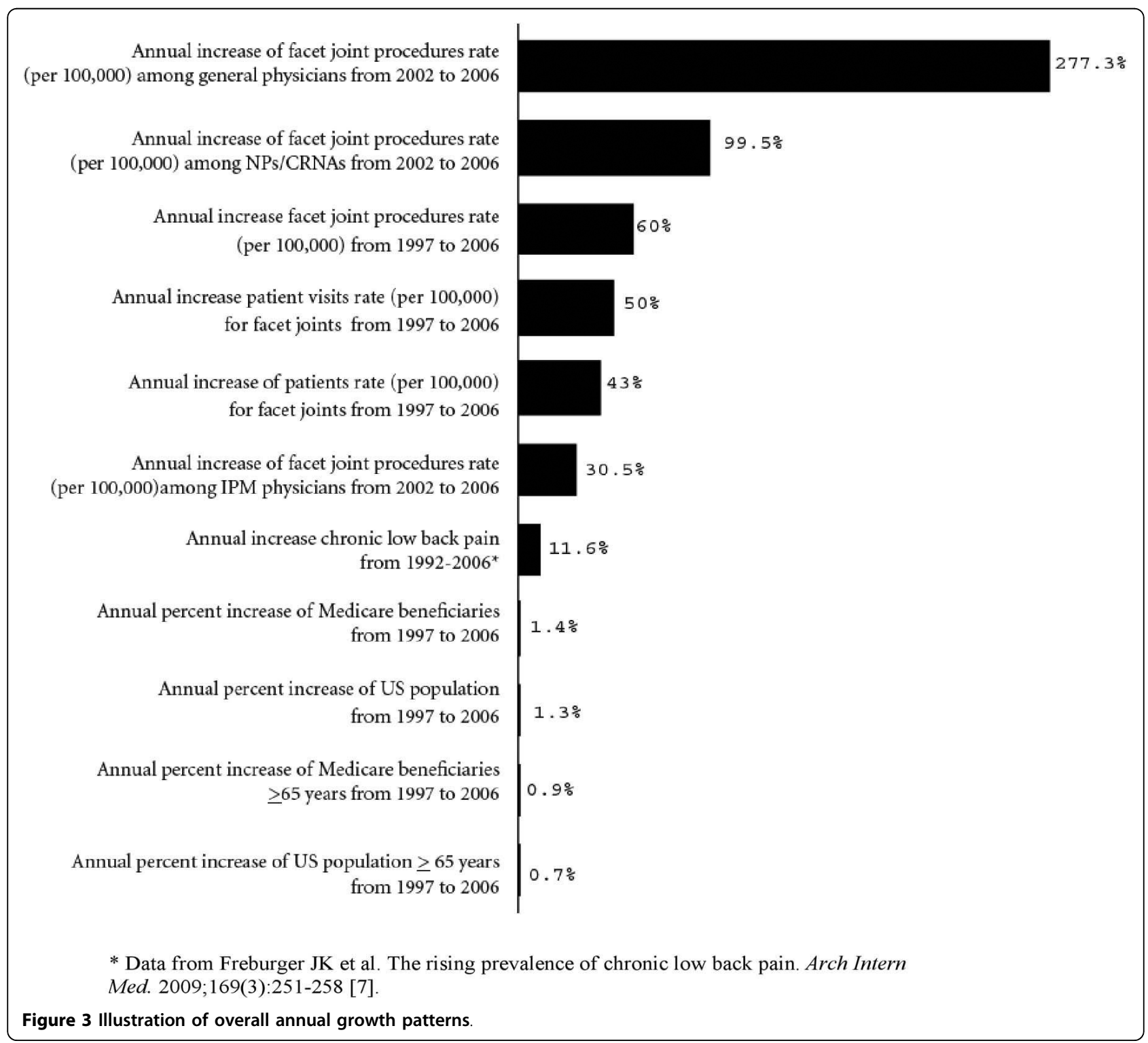


increases were substantially higher than any other specialty, even though overall increases were significant: $160 \%$ from 2002 to 2006, an annual increase of $40 \%$.

There was a 26.8-fold difference in the utilization pattern in Florida from Hawaii, the state with the lowest pattern for 2006. The remaining 49 states showed less than a 10 -fold difference. Further, it has been shown that $47 \%$ of facet joint interventions in Florida were performed by general physicians. There has been an exponential growth of facet joint interventions in office settings of $271 \%$ with ASC settings showing $168 \%$ growth and HOPD settings showing $40 \%$ growth. However, moving the procedures to hospital settings will not resolve the issue as the average cost of the total procedure in HOPD settings in 2006 was $\$ 467.80$, whereas in in-office settings, it was $\$ 227.60$ and in ASC settings, it was $\$ 352.20$.

Fluoroscopy utilization was lowest among family and general practice and internal medicine physicians and highest among pain management specialties. Non-fluoroscopically guided procedures present multiple issues regarding the accuracy of the procedure, medical necessity, and documentation.

With respect to evidence for facet joint interventions, there is emerging evidence to show the effectiveness of medial branch blocks and radiofrequency neurotomy along with effective diagnosis, when patients are selected appropriately meeting indications and medical necessity criteria [20-28]. While this evidence is emerging, some systematic reviews [19] have not utilized these trials [26-28] in their evidence synthesis.

Friedly et al [3] postulated that there was a disproportionate increase in procedures in ACSs, and that ACSs received higher payments. The implication is that these procedures had been shifted to ACSs as self referrals. Also that there was excessive use by facilitating physician investors to increase practice revenues by receiving facility payments for procedures. However, our study shows that this is not an issue. Rather, it may be due to the providing of more efficient services as a result of specialized staff and equipment, and convenient locations with short waiting times as well as better physician production. Further, the data illustrates that the procedures are more expensive in HOPD settings compared to ASC settings.

Based on the current data, it appears that the annual increase in the population with chronic low back pain is $11.6 \%$ [7], and the increase in facet joint intervention visits is approximately $50 \%$. The increases are much lower in states with stricter regulations and LCDs $[34,35]$. Kentucky showed an annual increase of $25 \%$ and Indiana, 26\%; whereas the annual increase in Florida was $95 \%$. The overall increase across the country was $40 \%$ from 2002 to 2006.
McKinsey Global Institute [30] postulated multiple factors for the increased growth of outpatient health care services in the United States. First, provider capacity growth and response to high outpatient margins is illustrated in this study based on significant increases in in-office settings and also performing these procedures. Other causes are that in outpatient settings, more efficient services are provided as a result of specialized staff and equipment, convenience of the location, short waiting times, and better physician production $[34,35]$. The second factor relates to judgment based on the nature of physician care. Over the years there has been significant growth in interventional pain management due to increased understanding and to the availability of a supply of physicians. The third factor described relates to technological innovation that drives prices higher rather than lower [36], which is not proven in this study in the Medicare population in the United States. The fourth factor relates to demand growth that appears to be due to the greater availability of supplies. While this is accurate, there is also demand due to access and also to the increasing prevalence of spinal pain. The final factor relates to relatively price-insensitive patients with limited out-of-pocket costs. This factor may be realistic in the overall health care evaluation. However, in the Medicare population, the application of this is minimal. In this study we included only the patients who were paying fee-for-service. Thus, price insensitivity does not apply. However, the study of the patients with third party insurance with low out-of-pocket costs and workers' compensation patients with no out-of-pocket costs and Medicare Advantage patients with low out-ofpocket costs or no out-of-pocket costs will illustrate these differences. Yet numerous problems continue to exist with overuse and abuse.

There are multiple limitations to our study. These include the lack of inclusion of participants in Medicare Advantage plans, which includes approximately $10 \%$ of enrollees, and potential coding errors [3,31]. However, we have included all patients over 65 receiving traditional fee-for-service Medicare and under 65 as well. This inclusion is important because patients below the age of 65 represent a significant proportion of patients receiving facet joint interventions, with a higher frequency of services. In general, patients less than 65 years of age received more intense and a higher proportion of services (504\% vs. 355\%) [2]. This fact is echoed in this evaluation, which shows an increase of facet joint services of $683 \%$ vs. $498 \%$ from 1997 to 2006 . Since the data does not contain HOPD facility charges, we had to estimate the facility charges for outpatient hospital charges, similar to Friedly et al [3]. Another limitation is that some variation may be related to coding errors and diagnostic ambiguity, and to non-reporting of 
fluoroscopy. However, due to the usage of actual data for physicians, ASCs, and office services, these errors should have very little influence.

Multiple recommendations have been made to slow the growth of health care costs in general and for interventional techniques in particular $[1,4,36]$. Health care experts have recommended policies that encourage high-growth or high-cost regions to behave more like slow-growth, low-cost regions and to encourage lowcost, slow-growth regions to sustain their current needs for interventional techniques to slow spending growth. The OIG [1] has recommended strengthening program efforts to prevent improper payments; others [3] have also recommended more stringent regulations on medical necessity, indications, accreditation provisions in the settings performed, and training and qualifications of the physicians performing the procedures.

\section{Conclusion}

In conclusion, our data summarizes the explosive growth of facet joint interventions in agreement with the OIG report [1] and other reports [2]. This review also demonstrates that the growth has been substantial in certain regions and by certain specialties. Some of the growth may be accounted for by improved access, precision of diagnostic and therapeutic modalities outcomes, and the increasing prevalence of spinal pain. However, there still continue to be multiple problems with ambiguity of diagnosis, lack of fluoroscopic use, disproportionate increase in procedures by some specialties and some regions, and escalating costs.

Additional file 1: Summary of the frequency of utilizations of various facet joint interventions in Medicare beneficiaries based on place of service in 2002 and 2006.

\section{Acknowledgements}

The authors wish to thank Sekar Edem for his assistance in the literature search and Tonie M. Hatton and Diane E. Neihoff, transcriptionists, for their assistance in the preparation of this manuscript.

\section{Author details}

'Pain Management Center of Paducah, 2831 Lone Oak Road, Paducah, KY, 42003, USA. ${ }^{2}$ Pain Diagnostics Associates, 1601 Roosevelt Road, Niagara WI 54151, USA. ${ }^{3}$ Texas Tech. University Health Sciences Center, 3601 4th St MS: 8182 Lubbock TX 79430, USA. ${ }^{4}$ Albany Medical College, 47 New Scotland Avenue, Albany NY 12208, USA. ${ }^{5}$ Massachusetts General Hospital, 55 Blossom St., Gray 289, Boston, MA 02114, USA.

\section{Authors' contributions}

$L M, V P, V S, M V B, H S S$, and JAH conceived the concept, design and coordination. VP processed the data. LM, VS, and MVB drafted the manuscript. HSS and JAH participated in the revision of the manuscript. All authors read and approved the final manuscript.

\section{Competing interests}

Dr. Manchikant is CEO and Chairman of American Society of Interventional Pain Physician representing interventional pain physicians across the nation; is Medical Director of the Pain Management Center of Paducah, the Ambulatory Surgery Center of Paducah, and Pain Care Surgery, providing interventional pain management services including facet joint interventions in an ambulatory surgery center and in an office setting; and is Associate Professor of Anesthesiology at The University of Louisville.

Mr. Pampati is a statistician and employed by Pain Management Center of Paducah. He is a non-physician and does not performing interventional techniques; however, he is involved extensively in statistical management of data related to interventional pain management.

Dr. Singh is Medical Director of the Pain Diagnostic Associates, Niagara, Wisconsin. He is an interventional pain management physician practicing in an ambulatory surgery center and in hospital outpatient departments including facet joint interventions.

Dr. Boswell is a Professor of Anesthesiology and Director of the International Pain Center, Texas Tech University Health Sciences Center, Lubbock, Texas. He practicing interventional pain management including Interventional techniques in all 3 settings; in hospital, hospital outpatient, and in-office settings.

Dr. Smith is an Associate Professor and Academic Director of Pain Management for Albany Medical College Department of Anesthesiology, Albany, New York. He practices interventional pain management including interventional techniques in hospital outpatient department setting. Dr. Hirsch is Chief of Minimally Invasive Spine Surgery with the Departments of Radiology and Neurosurgery, Massachusetts General Hospital; and an Associate Professor of Radiology, Harvard Medical School, Boston, Massachusetts. His practice also includes interventional radiology including interventional procedures, but does not include facet joint interventions.

Received: 4 November 2009 Accepted: 30 March 2010

Published: 30 March 2010

\section{References}

1. Department of Health and Human Services (DHHS). Office of Inspector General (OIG): Medicare Payments for Facet Joint Injection Services (OEI05-07-00200). 2008 [http://www.oig.hhs.gov/oei/reports/oei-05-07-00200. pdf], Accessed date: 8/3/2009.

2. Manchikanti L, Singh V, Pampati V, Smith HS, Hirsch JA: Analysis of growth of interventional techniques in managing chronic pain in Medicare population: A 10-year evaluation from 1997 to 2006. Pain Physician 2009, 12:9-34.

3. Friedly J, Chan L, Deyo R: Increases in lumbosacral injections in the Medicare population: 1994 to 2001. Spine 2007, 32:1754-1760.

4. Manchikanti L, Giordano J: Physician payment 2008 for interventionalists: Current state of health care policy. Pain Physician 2007, 10:607-626.

5. Manchikanti L, Boswell MV: Interventional techniques in ambulatory surgical centers: A look at the new payment system. Pain Physician 2007, 10:627-650.

6. Friedly J, Chan L, Deyo R: Geographic variation in epidural steroid injection use in Medicare patients. J Bone Joint Surg Am 2008, 90:1730-1737.

7. Freburger JK, Holmes GM, Agans RP, Jackman AM, Darter JD, Wallace AS, Castel LD, Kalsbeek WD, Carey TS: The rising prevalence of chronic low back pain. Arch Intern Med 2009, 169:251-258.

8. Boswell MV, Trescot AM, Datta S, Schultz DM, Hansen HC, Abdi S, Sehgal N, Shah RV, Singh V, Benyamin RM, Patel VB, Buenaventura RM, Colson JD, Cordner HJ, Epter RS, Jasper JF, Dunbar EE, Atluri SL, Bowman RC, Deer TR, Swicegood JR, Staats PS, Smith HS, Burton AW, Kloth DS, Giordano J, Manchikanti L: Interventional techniques: Evidence-based practice guidelines in the management of chronic spinal pain. Pain Physician 2007, 10:7-111.

9. Gureje O, Von Korff M, Simon GE, Gater R: Persistent pain and well-being: A World Health Organization Study in Primary Care. JAMA 1998, 280:147-151.

10. Bressler HB, Keyes WJ, Rochon PA, Badley E: The prevalence of low back pain in the elderly. A systemic review of the literature. Spine 1999, 24:1813-1819.

11. Côté P, Cassidy JD, Carroll L: The Saskatchewan Health and Back Pain Survey. The prevalence of neck pain and related disability in Saskatchewan adults. Spine 1998, 23:1689-1698. 
12. Luo X, Pietrobon R, Sun SX, Liu GG, Hey L: Estimates and patterns of direct health care expenditures among individuals with back pain in the United States. Spine 2004, 29:79-86.

13. Leigh JP, Markowitz SB, Fahs M, Shin C, Landrigan PJ: Occupational injury and illness in the United States. Estimates of costs, morbidity, and mortality. Arch Intern Med 1997, 157:1557-1568.

14. Manchikanti L, Singh V, Datta S, Cohen SP, Hirsch JA: Comprehensive review of epidemiology, scope, and impact of spinal pain. Pain Physician 2009, 12:E35-E70.

15. Manchikanti L, Boswell MV, Singh V, Benyamin RM, Fellows B, Abdi S, Buenaventura RM, Conn A, Datta S, Derby R, Falco FJE, Erhart S, Diwan S, Hayek SM, Helm S, Parr AT, Schultz DM, Smith HS, Wolfer LR, Hirsch JA: Comprehensive evidence-based guidelines for interventional techniques in the management of chronic spinal pain. Pain Physician 2009, 12:699-802.

16. Deyo RA, Mirza SK, Turner JA, Martin BI: Overtreating chronic back pain: Time to back off?. J Am Board Fam Med 2009, 22:62-68.

17. Edmond SL, Felson DT: Function and back symptoms in older adults. J Am Geriatr Soc 2003, 51:1702-1709.

18. Leveille SG, Guralnik JM, Hochberg M, Hirsch R, Ferrucci L, Langlois J, Rantanen T, Ling S: Low back pain and disability in older women: Independent association with difficulty but not inability to perform daily living activities. J Gerontol A Biol Sci Med Sci 1999, 54:M487-M493.

19. Staal JB, de Bie RA, de Vet HCW, Hildebrandt J, Nelemans P: Injection therapy for subacute and chronic low back pain. An updated Cochrane review. Spine 2009, 34:49-59.

20. Manchikanti L, Singh V, Derby R, Schultz DM, Benyamin RM, Prager JP, Hirsch JA: Reassessment of evidence synthesis of occupational medicine practice guidelines for interventional pain management. Pain Physician 2008, 11:393-482.

21. Boswell MV, Colson JD, Sehgal N, Dunbar EE, Epter R: A systematic review of therapeutic facet joint interventions in chronic spinal pain. Pain Physician 2007, 10:229-253.

22. Sehgal N, Dunbar EE, Shah RV, Colson JD: Systematic review of diagnostic utility of facet (zygapophysial) joint injections in chronic spinal pain: An update. Pain Physician 2007, 10:213-228.

23. Atluri S, Datta S, Falco FJE, Lee M: Systematic review of diagnostic utility and therapeutic effectiveness of thoracic facet joint interventions. Pain Physician 2008, 11:611-629.

24. Falco FJE, Erhart S, Wargo BW, Bryce DA, Atluri S, Datta S, Hayek SM: Systematic review of diagnostic utility and therapeutic effectiveness of cervical facet joint interventions. Pain Physician 2009, 12:323-344.

25. Datta S, Lee M, Falco FJE, Bryce DA, Hayek SM: Systematic assessment of diagnostic accuracy and therapeutic utility of lumbar facet joint interventions. Pain Physician 2009, 12:437-460.

26. Manchikanti L, Singh V, Falco FJE, Cash KA, Pampati V: Effectiveness of thoracic medial branch blocks in managing chronic pain: A preliminary report of a randomized, double-blind controlled trial; Clinical trial NCT00355706. Pain Physician 2008, 11:491-504.

27. Manchikanti L, Singh V, Falco FJ, Cash KA, Fellows B: Cervical medial branch blocks for chronic cervical facet joint pain: A randomized double-blind, controlled trial with one-year follow-up. Spine 2008 , 33:1813-1820.

28. Manchikanti L, Singh V, Falco FJ, Cash KA, Pampati V: Lumbar facet joint nerve blocks in managing chronic facet joint pain: One-year follow-up of a randomized, double-blind controlled trial: Clinical Trial NCT00355914. Pain Physician 2008, 11:121-132.

29. Hartman M, Martin A, McDonnell P, Catlin A, National Health Expenditure Accounts Team: National health spending in 2007: Slower drug spending contributes to lowest rate of overall growth since 1998. Health Aff (Millwood) 2009, 28:246-261.

30. Farrell D, Jensen E, Kocher B, Lovgrove N, Melhem F, Mendonca L, Parish B, McKinsey Global Institute: Medicare Enrollment: National Trends 1966 2007. Accounting for the Cost of US Health Care: A New Look at Why Americans Spend More McKinsey \& Company, McKinsey Global Institute 2008.

31. [http://www.cms.hhs.gov/MedicareEnRpts/Downloads/HISMI07.pdf], Accessed date: 8/3/2009.

32. Consumer Price Index. U.S. Department of Labor Bureau of Labor Statistics 2006, 3-25 [http://www.bls.gov/cpi/].
33. Providers Specialty codes, Appendix D. Medicare Part B Reference Manual. [http://www.highmarkmedicareservices.com/partb/refman/index. html], Accessed date: 8/3/2009.

34. Medicare Payment Advisory Commission: Report to the Congress: Paying for interventional pain services in ambulatory settings Washington, DC: MedPAC 2001.

35. Medicare Payment Advisory Commission: MedPac Report to Congress: Medicare Payment Policy. MedPac 2004, 185-204.

36. Fisher ES, Bynum JP, Skinner JS: Slowing the growth of health care costs lessons from regional variation. N Engl J Med 2009, 360:849-52.

\section{Pre-publication history}

The pre-publication history for this paper can be accessed here: http://www. biomedcentral.com/1472-6963/10/84/prepub

\section{doi:10.1186/1472-6963-10-84}

Cite this article as: Manchikanti et al.: Explosive growth of facet joint interventions in the medicare population in the United States: a comparative evaluation of 1997, 2002, and 2006 data. BMC Health Services Research 2010 10:84.

\section{Submit your next manuscript to BioMed Central and take full advantage of:}

- Convenient online submission

- Thorough peer review

- No space constraints or color figure charges

- Immediate publication on acceptance

- Inclusion in PubMed, CAS, Scopus and Google Scholar

- Research which is freely available for redistribution

Submit your manuscript at www.biomedcentral.com/submit
C) Biomed Central 